\title{
Long-Term Analysis of In Vivo Characteristics of Recording Electrode Using Electrochemical Impedance Spectroscopy
}

\author{
Mariko Kuwabara, ${ }^{1 *}$ Hiroyuki Tashiro, ${ }^{2}$ Yasuo Terasawa, ${ }^{1,3}$ Yukari Nakano, ${ }^{1,3}$ \\ Yurina Yoshimura, ${ }^{4}$ Koji Osawa,${ }^{5}$ Toshihiko Noda ${ }^{1}$ Takashi Tokuda, ${ }^{1}$ and Jun Ohta ${ }^{1}$ \\ ${ }^{1}$ Graduate School of Materials Science, Nara Institute of Science \& Technology \\ 8916-5 Takayama-cho, Ikoma, Nara 630-0192, Japan \\ ${ }^{2}$ Department of Health Sciences, Faculty of Medical Sciences, Kyushu University \\ 3-1-1 Maidashi, Higashi-ku, Fukuoka 812-8582, Japan \\ ${ }^{3}$ Artificial Vision Institute, Research and Development Division, Nidek Co., Ltd. \\ 13-2 Hama-cho, Gamagori, Aichi 443-0036, Japan \\ ${ }^{4}$ Department of Health Sciences, Graduate School of Medical Sciences, Kyushu University \\ 3-1-1 Maidashi, Higashi-ku, Fukuoka 812-8582, Japan \\ ${ }^{5}$ Artificial Retina Development Office, Development Division, Nidek Co., Ltd. \\ 34-14 Maehama Hiroishi-cho, Gamagori, Aichi 443-0038, Japan
}

(Received May 1, 2017; accepted September 14, 2017)

Keywords: electrochemical impedance spectroscopy (EIS), equivalent circuit, emulation, visual evoked potential (VEP), long-term recording

Implanted electrodes are widely used for in vitro and in vivo neural recording; however, the established properties of these electrodes can change over time. Here, we characterize the longterm changes in the electrode surface during in vivo recording and investigate the effect of these changes on visual evoked potentials (VEPs) using electrochemical impedance spectroscopy (EIS) spectra. VEP emulation was simulated on the basis of EIS measurements to identify the factors that contribute to time-dependent changes in the VEP. The VEP measurements revealed a timedependent decrease in the latency for the first negative peak, while the amplitude was stable. EIS measurements revealed a stable impedance. An equivalent circuit analysis confirmed a timedependent increase in solution resistance. However, VEP emulation indicated that the VEP changes were not related to changes in the recording electrode properties, but rather, to changes in vital reactions. Our results suggest that time-dependent VEP measurement requires EIS and emulation analyses to identify changes in the characteristics of the interface between the recording electrode surface and the surrounding tissue and the effects of these changes on electrical recordings.

\section{Introduction}

Microelectrode arrays (MEAs) and implanted electrodes are routinely used for in vitro and in vivo recording of neuronal electric potentials. ${ }^{(1,2)}$ Notably, implanted electrodes are also used in therapeutic devices such as cochlear implants and deep brain stimulators for both nerve stimulation and nerve response recording. ${ }^{(3,4)}$ Electrode impedance can affect the recorded nerve activity ${ }^{*}$ Corresponding author: e-mail: kuwabara.mariko.kd8@ms.naist.jp http://dx.doi.org/10.18494/SAM.2017.1635 
wave pattern; thus, the development of such devices and treatment strategies must consider such electrode properties. ${ }^{(5)}$

The development of novel implantable neural devices and treatment strategies can also involve the use of in vivo implanted electrodes for chronic recordings. We developed a prosthetic to restore lost vision through the electrical stimulation of the remaining optic nerve in patients with blindness owing to retinitis pigmentosa or age-related macular degeneration. ${ }^{(6)}$ To evaluate the safety of our device, brain-implanted recording electrodes were used for the chronic recording of visual evoked potentials (VEPs). ${ }^{(7)}$ The assessment of chronic VEP recordings can also be applied to the practical research of ophthalmological medicines, such as the protective medication of the optic nerve. ${ }^{(8)}$ Other studies involving evoked potential recordings include that by Wang et al. who chronically recorded evoked potentials using an MEA. ${ }^{(9)}$ Notably, Guarino et al. reported that anesthesia conditions and the recording electrode position can affect the recording of evoked potentials, ${ }^{(10)}$ and similarly, Charng et al. reported the effects of anesthesia on wireless VEP measurement. ${ }^{(1)}$

A time-dependent and functional evaluation of the optic nerve using chronic VEP measurement requires an understanding of the factors that affect the VEP. During chronic measurement of evoked potentials under a fixed anesthetic depth, the acquired waveform may change in response to several factors. For example, such variations may be related to changes in not only stimulation parameters, but also vital reactions, recording electrode properties, and the surrounding tissue environment of the recording electrode. ${ }^{(2)}$

To accurately evaluate time-dependent functional changes of the optic nerve, we developed recording electrodes that minimize brain tissue damage, ${ }^{(7)}$ and successfully obtained chronic VEP recordings using our electrodes. However, we observed a time-dependent decrease in the first negative wave $\left(\mathrm{N}_{1}\right)$ latency of the VEP. ${ }^{(7)}$ Identifying the causes of VEP changes using only an evoked potential analysis can lead to false positive results. To identify changes in the characteristics of the interface between the electrode and the surrounding tissue, an analysis of the time-dependent recording electrode characteristics using electrochemical impedance spectroscopy (EIS) may be useful. ${ }^{(12)}$ EIS is a nonsteady assay for determining electrode reaction mechanisms using a frequency response analysis that involves sine wave potential. With this analysis, the frequency response of the electrode impedance is expressed in a Bode plot or Cole-Cole plot. Bode plots can be used to express the magnitude of the impedance and phase shift against the logarithm of the frequency. Cole-Cole plots can be used to present the locus of the impedance response against each frequency in the complex plane. ${ }^{(13)}$ Nonetheless, these plots are difficult to use to determine the behavior of the electrode.

Equivalent circuit analyses using minimal simplified elements are useful for expressing electrode behavior. Equivalent circuit analysis yields parameters that reflect physical activity, and thus are useful for EIS interpretation. In a previous study, an impedance of $1 \mathrm{kHz}$ was used to evaluate chronic changes in the properties of a recording electrode. ${ }^{(14)}$ In this study, we found that electrode frequency impedance is related to the capacitive property of the electrode, but not to other characteristics, such as the reactivity of the electrode or the solution resistance. ${ }^{(5)}$ Various parameters of equivalent circuit analysis can be used to understand the surface interface behavior of an electrode. ${ }^{(15)}$ Equivalent circuit analysis has been applied in several in vivo and in vitro studies. ${ }^{(16-18)}$ For example, short-term changes in the properties of in vivo recording electrodes have been reported. ${ }^{(5)}$ However, long-term changes in the properties of in vivo recording electrodes are unknown. In addition, modulation of the evoked potentials due to such changes in the 
properties of the recording electrode surface interface has not been investigated using equivalent circuit analyses. As aforementioned, such time-dependent changes in chronically recorded evoked potentials may occur in response to physiological stimulation or recording electrode characteristics.

Electrical circuit models for cortical recordings have been proposed. ${ }^{(19,20)}$ Emulation analyses of such models and simulation of neuron activity well describe potential waveforms and amplitudes. $^{(21)}$ A simple method can also be used to simulate electroencephalography (EEG) data. ${ }^{(22,23)}$ Although the effect of a change in electrode interface activity on time-dependent evoked potentials is unknown, these methods for emulation and simulation may validate models that predict a relationship between the evoked potentials and the environment surrounding the recording electrode. Although chronic evoked potentials have been recorded using the implanted electrodes,${ }^{(9-11)}$ there are no reports using chronic VEP recordings and EIS measurement, equivalent circuit analysis, and VEP emulation in a single study.

In this study, we investigate whether the changes in the VEP result are related to the in vivo electrode properties, the tissue environment surrounding the recording electrode, or other factors. To investigate the recording electrode properties, we used EIS. Clarifying the cause of VEP variation will allow for a distinction between changes in the VEP, which result from the degeneration of either the optic nerve or the recording electrode.

\section{Materials and Methods}

\subsection{Electrode design}

We developed recording electrodes that were designed to minimize mechanical stress to brain tissue. $^{(7)}$ The electrodes were implanted into three Dutch rabbits (weight $2.6 \mathrm{~kg}$, age 19 weeks), hereafter referred to as 'rabbit A', 'rabbit B', and 'rabbit C'. Platinum (Pt) ball-tip screw electrodes were implanted into rabbit A and rabbit B. The Pt ball-tip screw electrode had the structure of a plastic screw and was composed of polymethylmethacrylate with Pt balls on the tip [Fig. 1(a)]. ${ }^{(7)}$ Smooth-surface Pt balls were constructed of Pt wire (351265, Nilaco, Tokyo, Japan). The length of the electrode was $17.5 \mathrm{~mm}$, the diameter of the screw was $2.6 \mathrm{~mm}$, and the diameter of the Pt balls was $0.7 \mathrm{~mm}$. The threaded insert made of stainless steel (Ensat 302000025.500, KKV, Amberg, Germany) was used to adjust the insertion depth of the electrode into the brain to enable the optimization of the VEP. Hereafter, the platinum ball-tip screw electrode is referred to as the recording SE. The platinum/iridium (Pt/Ir) ball-tip planar MEA that was implanted into rabbit $\mathrm{C}$ had a sheet structure with Pt/Ir ball electrodes arranged in a $3 \times 3$ lattice pattern [Fig. 1(b)]. ${ }^{(7)}$ The MEA was composed of a Pt/Ir wire (9 twist lines; 967223, Nilaco), silicone sheets ( $0.1 \mathrm{t}$ and $0.2 \mathrm{t}$, Unique Medical, Tokyo, Japan), a silicone tube (986902, AS ONE, Tokyo, Japan), silicone adhesive (KE-41-T, Shin-Etsu Chemical, Tokyo, Japan), and a connector (HR25-9TR-12P, Hirose Electric, Tokyo, Japan). The sheet size was approximately $8.3 \times 12 \mathrm{~mm}^{2}$. The diameter of the $\mathrm{Pt} / \mathrm{Ir}$ ball was approximately $0.7 \mathrm{~mm}$. Hereafter, the Pt/Ir ball-tip planar MEA is referred to as the recording MEA. The recording SEs were implanted $6 \mathrm{~mm}$ anterior and $6 \mathrm{~mm}$ lateral to the lambda suture. The recording MEA was inserted under the right skull. Indifferent electrodes (M2 stainless steel anchor screw AM2-8, Unique Medical) were placed $14 \mathrm{~mm}$ anterior to the lambda suture. Additional details of the recording electrodes have been published. ${ }^{(7)}$ 


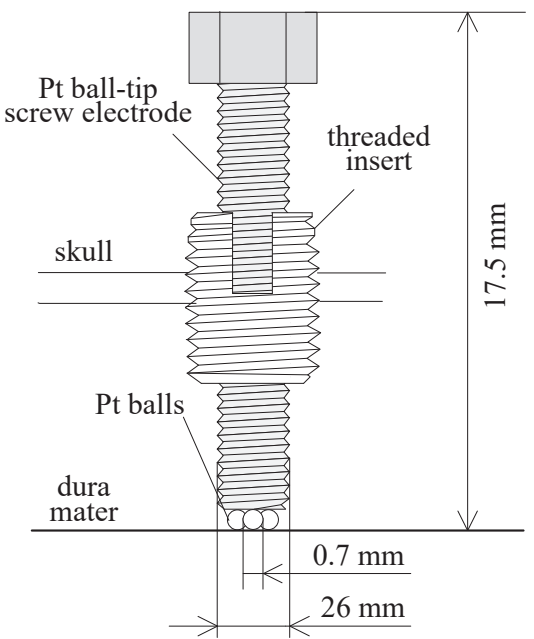

(a)
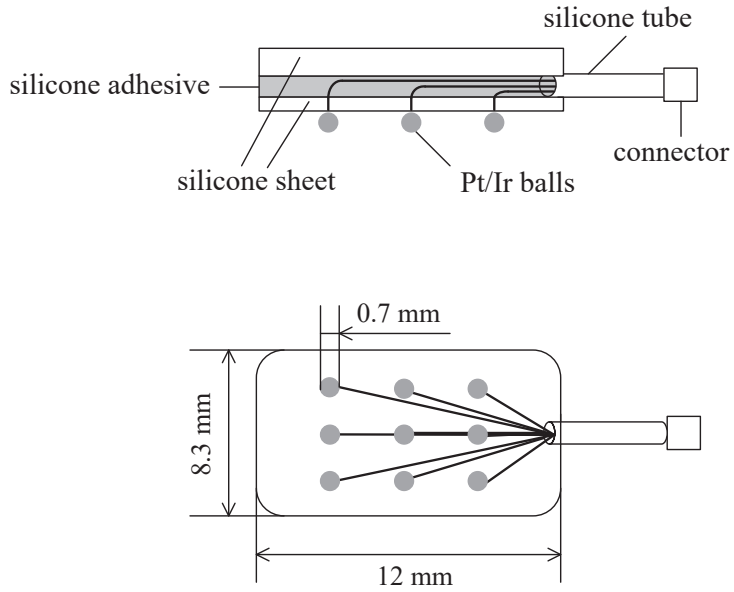

(b)

Fig. 1. Schematic view of the recording electrodes. (a) Pt ball-tip screw electrode. This electrode was fabricated from a plastic screw made of polymethylmethacrylate and Pt balls on the tip. The insertion depth of electrode into the brain was adjusted using the threaded insert to optimize the VEP recording. (b) Pt/Ir ball-tip planar MEA. This electrode had a silicone sheet structure with $\mathrm{Pt} / \mathrm{Ir}$ ball electrodes arranged in a $3 \times 3$ lattice pattern.

\subsection{VEP recording}

Each rabbit was fasted for $2 \mathrm{~h}$ before beginning the experiment. A rabbit was then placed in a recovery box that was covered with black-out curtains and connected to an anesthesia apparatus (NS-3000, Acoma Medical, Tokyo, Japan). Next, oxygen at a flow rate of $4 \mathrm{~L} / \mathrm{min}$ was introduced into the recovery box. The laboratory lights were turned off, and the rabbit was adapted to the dark for $15 \mathrm{~min}$. Subsequently, vaporized isoflurane gas (Forane, AbbVie, North Chicago, USA) was introduced into the recovery box until a $2.5 \%$ concentration was reached in the box while confirming with a medical monitor (BP-608EV, Nihon Colin, Tokyo, Japan). Once a sufficient anesthetic depth was reached, the anesthesia was switched to mask anesthesia at a $2.4 \%$ anesthetic concentration with an oxygen flow rate of $1 \mathrm{~L} / \mathrm{min}$. The pupil of the stimulated eye was dilated using tropicamide (Mydrin-P, Santen, Osaka, Japan). Local anesthesia (oxybuprocaine hydrochloride; Benoxil, Santen) and a drying agent (Scopisol, Senju, Osaka, Japan) were then applied. The stimulated eye was opened using an eye-opening device 5 min after applying local anesthesia. The contralateral eye was covered with gauze after the application of a drying agent to prevent dryness. The luminance of the room was maintained at $0.2-0.31 \mathrm{x}$. During the experiment, the anesthetic depth was controlled by adjusting the $2.4 \%$ end tidal concentration of the isoflurane gas based on the medical monitor information. The experiment began after the isoflurane concentration of the expired gas was stable for $5 \mathrm{~min}$. Light stimuli with a $20 \mathrm{~J}$ light intensity and 3 s repetition period from a flash stimulator (SLS-3100, Nihon Kohden, Tokyo, Japan) were adapted at a distance of $30 \mathrm{~cm}$ from the eyes. The actual measured values of the flash stimuli were as follows: $3.74 \mathrm{~cd} \cdot \mathrm{s} / \mathrm{cm}^{2}$ for the time-integrated luminance, $29.5 \mathrm{~cd} / \mathrm{cm}^{2}$ for the peak luminance, and $75 \mathrm{~ms}$ for the half-value width with respect to the peak luminance. Evoked potentials were recorded using a biological signal recording analysis system (PowerLab/8 SP, 
ML135, AD Instrument, Sydney, Australia). Recording was carried out at frequencies of 1-100 $\mathrm{Hz}$ and 32 counts on average. The latency and amplitude measured using the peak-to-trough method for $\mathrm{N}_{1}$ were evaluated. ${ }^{(24)}$ VEPs were recorded over a 32-week period after the electrode implantation.

\subsection{EIS analysis}

The EIS analysis was performed on rabbits A and B in which the recording SE was implanted. The EIS spectra using the three-electrode method were measured using a potentiostat equipped with a frequency response analyzer (FRA) (Autolab PGSTAT32, Metrohm, Herisau, Switzerland). The working electrode (WE) terminal was connected to the Pt-constructed recording SE, the counter electrode (CE) terminal was connected to the stainless steel-constructed indifferent electrode, and the reference electrode (RE) terminal was connected to the silver/silver chloride (Ag/ $\mathrm{AgCl})$-constructed ear clip electrode. The measurement conditions were a frequency of 1-1000 Hz and an applied voltage of $1 \mathrm{mV}_{\text {rms }}$ with a single sine constant voltage. In vitro EIS was measured before implantation in the $0.01 \mathrm{~mol} / \mathrm{L}$ phosphate-buffered saline (164-18541, Wako Chemicals, Osaka, Japan) with $0.2 \mathrm{~g} / \mathrm{L}$ albumin (020-01862, Kishida Chemical, Osaka, Japan) at $37^{\circ} \mathrm{C}$.

\subsection{Equivalent circuit analysis}

The EIS measurements were analyzed using electrochemical analysis software (NOVA, Metrohm). The circuit shown in Fig. 2 was used for the equivalent circuit analyses and was previously published as an electrode interface equivalent circuit. ${ }^{(25)}$ The solution resistance $\left(R_{s o l}\right)$ was the bulk resistance of the solution. ${ }^{(25)}$ The charge transfer resistance $\left(R_{c t}\right)$ indicated the difficulty of the charge transfer between the electrode and the electrolyte solution interface. ${ }^{(5)}$ The constant phase element (CPE) approximated an electric double-layer capacitor with a nonlinear characteristic. A deviation from an ideal capacitance behavior corresponds to a frequencydependent phase angle and the CPE represents the nonideal behavior as a complex impedance having the special characteristic of a phase angle that is independent of frequency. The capacitance dispersion at solid electrodes strongly depends on the electrode surface state, e.g., its surface roughness, the degree of crystallinity, and anion adsorption. ${ }^{(26)}$ The impedance of the CPE $\left(Z_{C P E}\right)$ is defined as Eq. (1). The CPE functions as a complete capacitor when $n=1$ and as a complete resistor when $n=0 .{ }^{(5)}$ The index $Y_{0}$ equals the admittance of the CPE at the angular frequency $\omega=$ $1 \mathrm{rad} / \mathrm{s}$.

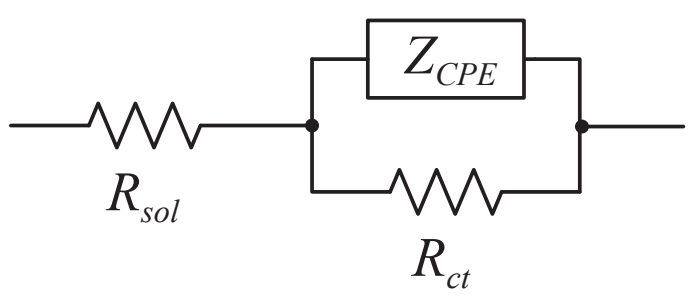

Fig. 2. Equivalent circuit model for the recording electrode $\left(R_{\text {sol }}\right.$ : solution resistance, $R_{c t}$ : charge transfer resistance, and $Z_{C P E}$ : impedance of constant phase element). 


$$
Z_{C P E}=\frac{1}{Y_{0}(j \omega)^{n}}
$$

The equivalent circuit was analyzed using a frequency range of $5-1000 \mathrm{~Hz}$, which is the frequency component of the $\mathrm{N}_{1}$ response. ${ }^{(27)}$

\subsection{VEP emulation}

The evoked potential was emulated using circuit simulation software (LTspice, Linear Technology, Milpitas, CA, USA). The equivalent circuit of the electrode interface is depicted in Fig. 3. To simulate brain tissue in the circuits, we used a parallel circuit with bulk tissue resistance (1480 $\Omega)$ and tissue capacitance $\left(2.99 \times 10^{-7} \mathrm{~F}\right){ }^{(20)}$ The CPE in the equivalent circuit can approximate capacitor activity. The input impedance of the bioamplifier (ML135, AD Instrument) consisted of a parallel circuit with an input resistor $(200 \mathrm{M} \Omega)$ and an input capacitor (200 pF). The signal current source as the neural response was defined as Eq. (2). ${ }^{(22)}$

$$
I(t)=\frac{1}{\sqrt{2 \pi \sigma}} \exp \left[\frac{-\left(t-t_{c}\right)^{2}}{2 \sigma^{2}}\right]
$$

Here, $t_{c}$ represents the start time for the activation of the neurons and $\sigma$ represents the duration of the activation. $I(t)$ is expressed in units of $\mathrm{A}$, and $t_{c}$ and $\sigma$ are expressed in units of $\mathrm{s}$. The values of $t_{c}$ and $\sigma$ were calculated on the basis of the VEP waveform at 8 weeks after electrode implantation. In our study, $t_{c}$ refers to the actual measured $\mathrm{N}_{1}$ latency and $\sigma$ is calculated on the basis of the actually measured duration of the $\mathrm{N}_{1}$ peak. The amplitude of $I(t)$, as shown in Eq. (2), is corrected with the conformation of the emulated evoked-potential $V_{\text {in }}$ illustrated in Fig. 3 to fit the actual

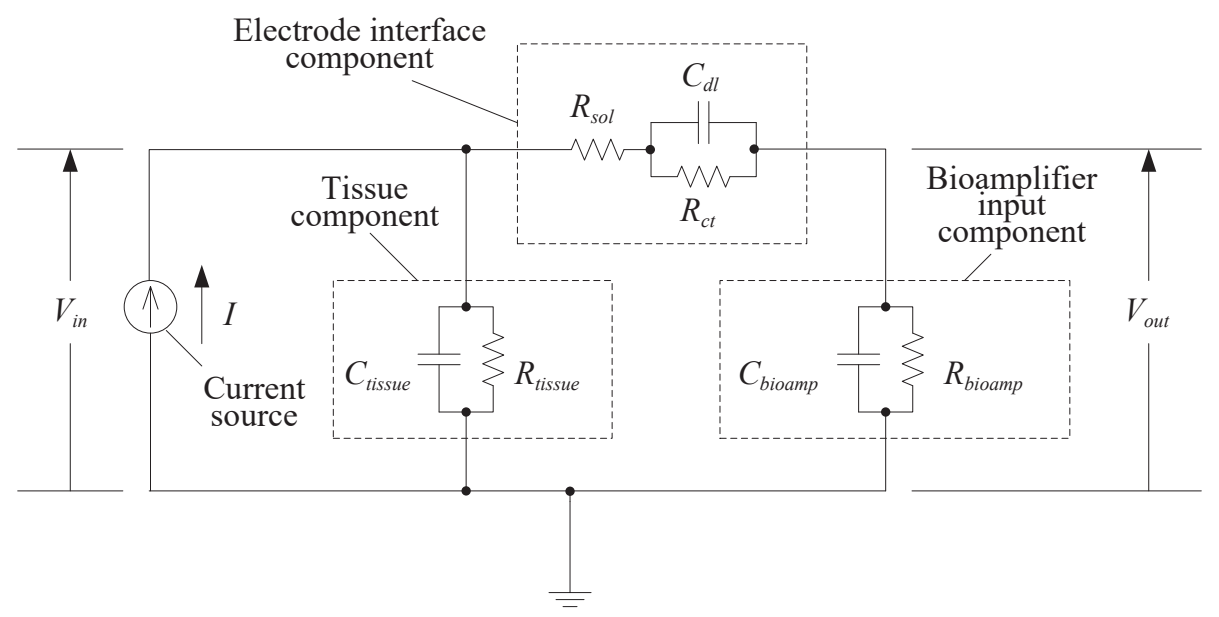

Fig. 3. An equivalent circuit to emulate the first negative wave of the visual evoked potential $\left(R_{\text {tissue }}\right.$ : tissue resistance, $C_{t i s s u e}$ : tissue capacitance, $R_{\text {sol }}$ : solution resistance, $R_{c t}$ : charge transfer resistance, $C_{d l}$ : double-layer capacitance, $R_{\text {bioamp }}$ : input resistance of bioamplifier, and $C_{\text {bioamp }}$ : input capacitance of bioamplifier). The current source $I$ emulates an electrical source model for the evoked potential, as denoted in Eq. (3). $V_{\text {in }}$ represents the electrical potential on the surface of the recording electrode. $V_{\text {out }}$ is input voltage to the bioamplifier after electrode interface modulation. 
VEP that was measured at 8 weeks after implantation. Thereafter, the signal current source in the emulation is represented as Eq. (3).

$$
I(t)=-4.34 \times 10^{-7} \times \exp \left[\frac{-(t-0.0265)^{2}}{10^{-5}}\right]
$$

For this emulation, the equivalent circuit analysis results of rabbit A were used. The values for $R_{c t}$ and the capacitor-approximated CPE were based on the value measured at 8 weeks after electrode implantation. The regulation value for solution resistance was on the basis of the value measured at 8 weeks after electrode implantation. This value was compared with the maximum value at 32 weeks after implantation. In addition, emulation was attempted using an extremely high value of solution resistance (10 M $\Omega$ ). The simulation values for VEP emulation are shown in Table 1. The results obtained from the circuit simulation software were filtered using a range of $1-100 \mathrm{~Hz}$.

The animal experiment was performed in compliance with the guidelines of the Association for Research in Vision and Ophthalmology regarding the use of animals in ophthalmic and visual research. Research approval was also obtained from the Animal Experimentation Ethics Committee at Nidek.

\section{Results}

Figure 4 shows the typical VEPs obtained at 8 weeks after the electrode implantation. The parameters recorded from each rabbit all showed similar values. Figures 5 and 6 show timedependent changes in the amplitude and latency of $\mathrm{N}_{1}$ from the VEPs in Fig. 4. A significant correlation was observed between latency and the electrode implantation period from week 6 to 32 (rabbit A: correlation coefficient $r=-0.85$, test of non-correlations, $p<0.01, t=4.26$; rabbit B: $r=-0.82, p<0.01, t=3.73$; rabbit $\mathrm{C}: r=-0.92, p<0.01, t=6.24)$. These data indicate that latency was reduced over time. Moreover, amplitude was stable and there was no correlation between amplitude and the week 12 to 32 period of electrode implantation (rabbit A: correlation coefficient $r=0.01$, test of non-correlations, $p>0.05, t=0.02$; rabbit B: $r=-0.26, p>0.05, t=0.54$; rabbit $\mathrm{C}$ : $r=0.28, p>0.05, t=0.57)$.

Table 1

Simulation values of visual evoked potential emulation parameter.

\begin{tabular}{lcc}
\hline Parameter & Description & Simulation value \\
\hline$R_{\text {tissue }}$ & Tissue resistance & $1480 \Omega$ \\
$C_{\text {tissue }}$ & Tissue capacitance & $299 \mathrm{nF}$ \\
$R_{\text {sol }}(8$ weeks; & Solution resistance $(8$ weeks; & $1415 \Omega ;$ \\
$\quad$ maximum; & maximum in 32 weeks; & $1757 \Omega ;$ \\
$\quad$ extremely high value $)$ & extremely high value $)$ & $10 \mathrm{M} \Omega$ \\
$R_{c t}$ & Charge transfer resistance & $1.1 \mathrm{~T} \Omega$ \\
$C_{d l}$ (approximated CPE) & Double-layer capacitance & $217 \mathrm{nF}$ \\
$R_{\text {bioamp }}$ & Input resistance of bioamplifier & $200 \mathrm{M} \Omega$ \\
$C_{\text {bioamp }}$ & Input capacitance of bioamplifier & $200 \mathrm{pF}$ \\
\hline
\end{tabular}




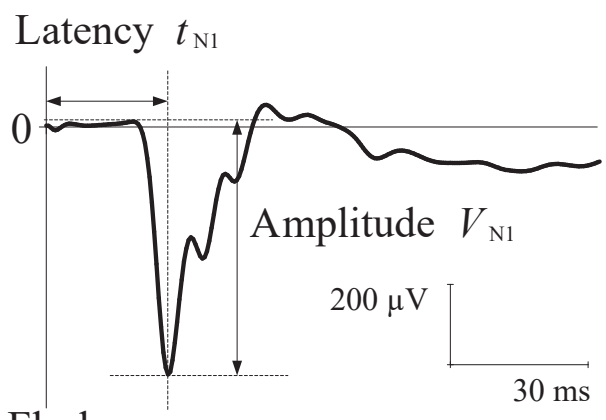

Flash

Fig. 4. A typical waveform of the visual evoked potential at 8 weeks after electrode implantation. Flash stimulation was applied at time zero.

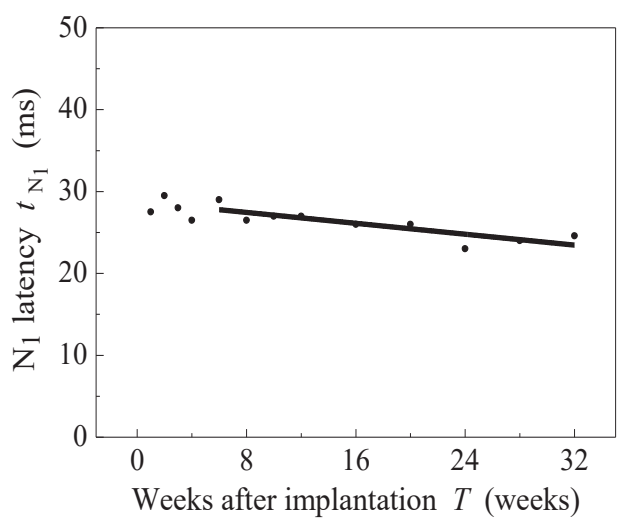

Fig. 5. Changes in the $\mathrm{N}_{1}$ latency of the visual evoked potential over time. ${ }^{(7)}$ The solid line was obtained using a least-squares method with data collected at 6-32 weeks after electrode implantation. The latency period appeared to decrease with time.

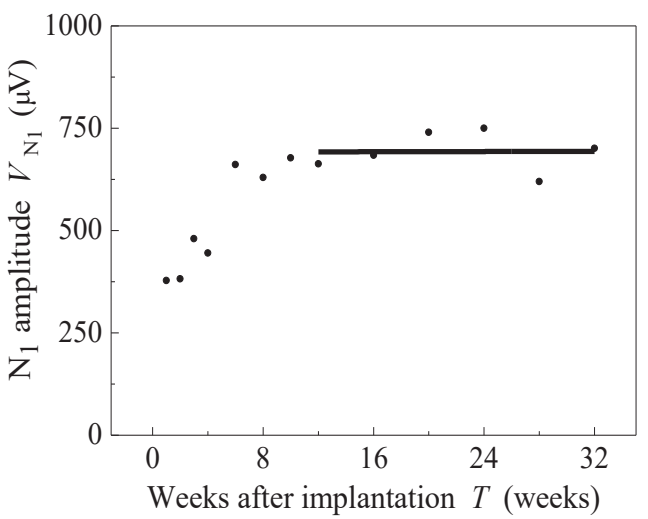

Fig. 6. Changes in the $\mathrm{N}_{1}$ amplitude of the visual evoked potential over time. ${ }^{(7)}$ The solid line was obtained using a least-squares method with data collected at 12-32 weeks after electrode implantation. Except for a few weeks after electrode implantation, over the 32-week period, the $\mathrm{N}_{1}$ amplitude was stable.

The electrochemical impedances were stable over time at each frequency after week 6 of electrode implantation (Fig. 7). The Cole-Cole plot and Bode plot at week 8 of electrode implantation are shown in Figs. 8 and 9, respectively. The results from the equivalent circuit simulation were consistent with the experimental findings (Figs. 8 and 9). The equivalent circuit analysis was based on the equivalent circuit illustrated in Fig. 2 and the results of the analysis are shown in Figs. 10-13. A significant correlation was found between $R_{\text {sol }}$ and the week 4 to 32 period of electrode implantation (rabbit A: correlation coefficient $r=0.68$, test of non-correlations, $p<0.05, t=2.63$; rabbit B: $r=0.65, p<0.05, t=2.43$ ). These results indicate that $R_{\text {sol }}$ increased over time (Fig. 10). In addition, in vitro $R_{\text {sol }}$ was significantly lower than the in vivo value. $R_{c t}$ gradually increased during the few weeks after electrode implantation and then reached infinity (Fig. 11). The CPE admittance was stable at 8 weeks after electrode implantation (Fig. 12). The value of 


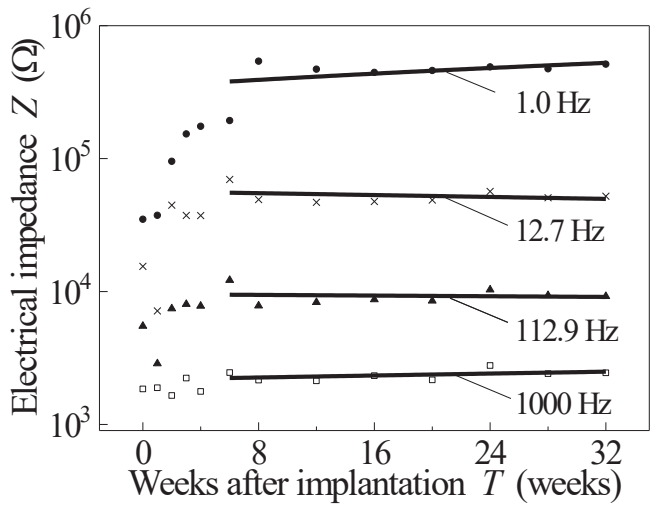

Fig. 7. Change in electrical impedance of the constructed electrodes over time. ${ }^{(7)}$ The solid lines were obtained using a least-squares method with data collected at 6-32 weeks after electrode implantation. Except for a few weeks after electrode implantation, over the 32-week period, the electrical impedances were stable.

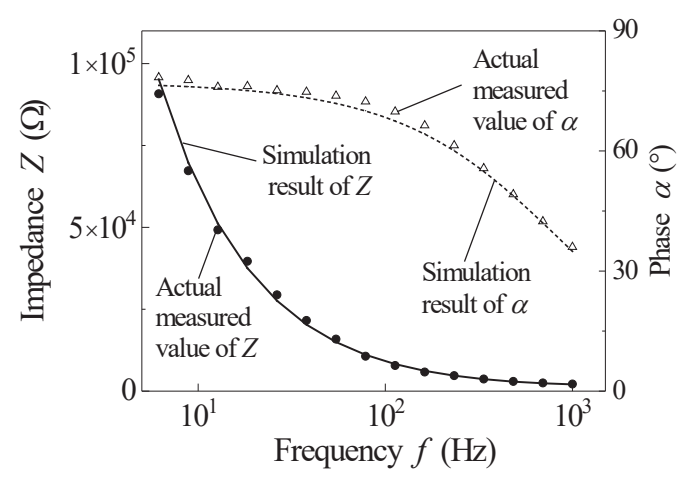

Fig. 9. Result of electrochemical impedance spectroscopy (Bode plot) at 8 weeks after electrode implantation. The plotted points indicate actual measured values and the lines represent the simulation results from the equivalent circuit analysis.

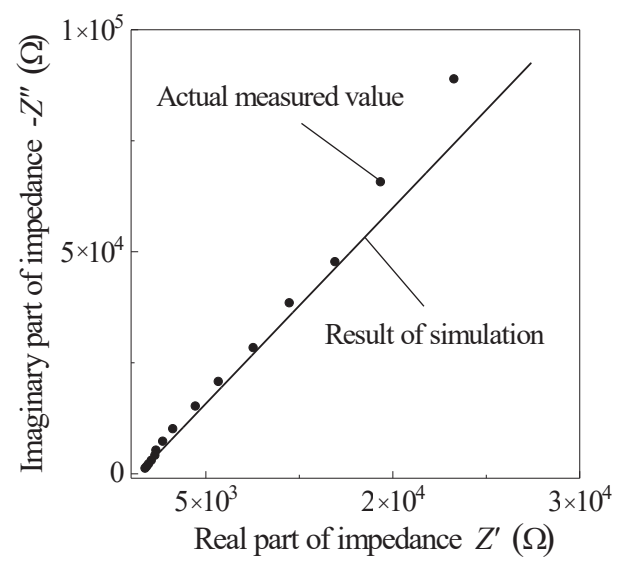

Fig. 8. Result of electrochemical impedance spectroscopy (Cole-Cole plot) at 8 weeks after electrode implantation. The plotted points indicate actual measured values and the line represents the simulation result from the equivalent circuit analysis.

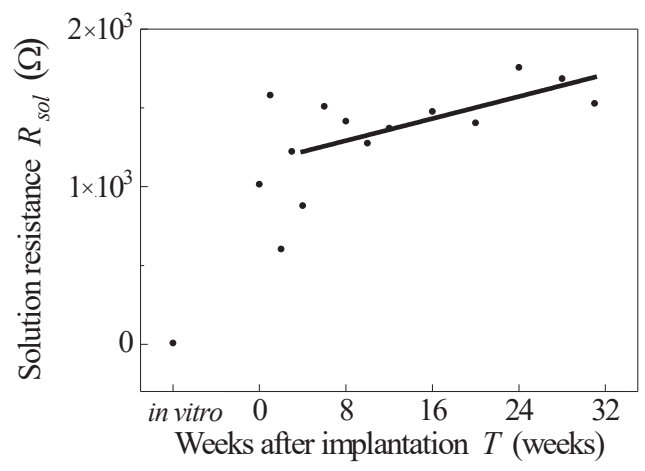

Fig. 10. Time-dependent changes in in vivo solution resistance $R_{\text {sol }}$. 'In vitro' denotes the value measured in phosphate-buffered saline before implantation. The solid line was obtained using a least-squares method with data collected at 4-32 weeks after electrode implantation. A positive correlation between $R_{\text {sol }}$ and time is apparent.

$n$ in Eq. (1) was also stable and close to 1 (Fig. 13). The in vivo $R_{c t}$ and CPE values stabilized at a level close to the in vitro values after several weeks of electrode implantation.

The results of the VEP emulation are shown in Figs. 14-16. The values of the parameters, which were from rabbit A, are shown in Table 1. Figures 14 and 15 show the results of the VEP emulation using the regulation value from week 8 for $R_{\text {sol }}$ and the maximum value during the 32-week period for $R_{\text {sol }}$, respectively. Figure 16 shows the results of the VEP emulation using significantly higher $R_{\text {sol }}$ values than those obtained in the actual animal experiment. The emulation results based on 


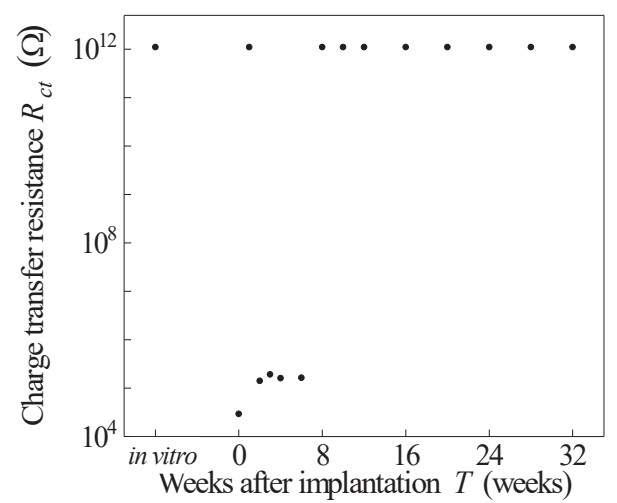

Fig. 11. Time-dependent changes in in vivo charge transfer resistance $R_{c t}$. 'In vitro' denotes the value measured in phosphate-buffered saline before implantation. A trend of a gradual increase was observed during the few weeks after electrode implantation. The value of $R_{c t}$ then reached infinity.

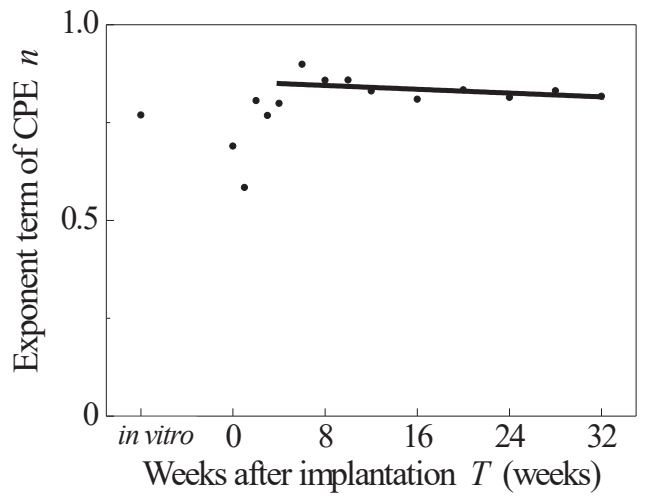

Fig. 13. Time-dependent changes in the exponent term $n$ of the in vivo CPE. 'In vitro' denotes the value measured in phosphate-buffered saline before implantation. The solid line was obtained using a least-squares method with data collected at 4-32 weeks after electrode implantation. Except for a few weeks after implantation, over the 32-week period, the values were stable and close to 1 .

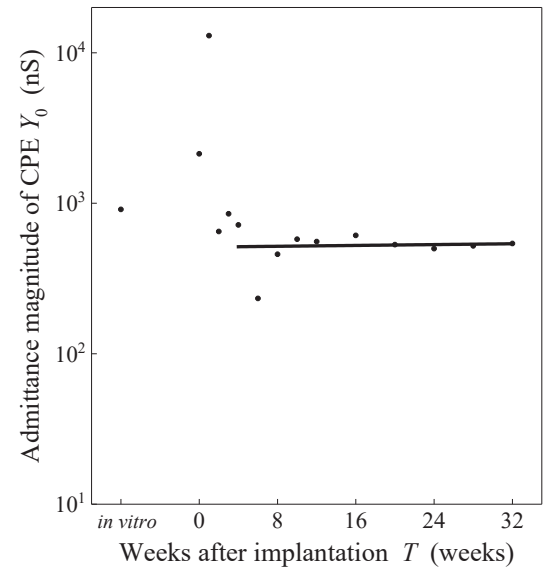

Fig. 12. Time-dependent changes in the magnitude of the in vivo admittance $Y_{0}$ of the CPE. 'In vitro' denotes the value measured in phosphate-buffered saline before implantation. The solid line was obtained using a least-squares method with data collected at 4-32 weeks after electrode implantation. Over the 32-week period, the values were stable, except for a few weeks after electrode implantation.

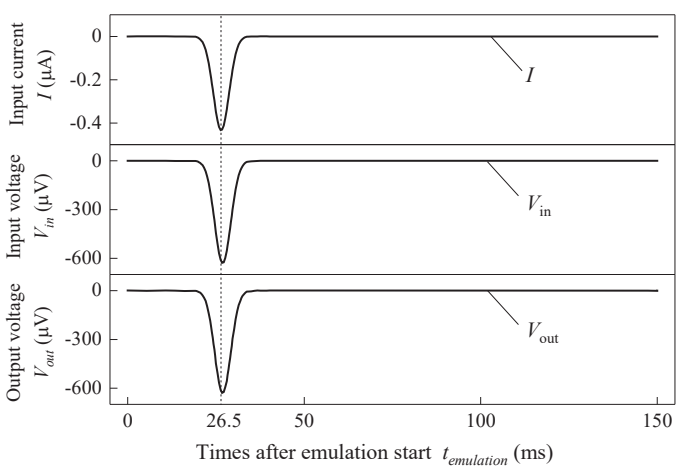

Fig. 14. Result of visual evoked potential emulation with the standard (8-week) value for solution resistance in rabbit A. The input current $I$ is denoted in Eq. (3). The input voltage $V_{\text {in }}$ and the output voltage $V_{\text {out }}$ are as described in Fig. 2. Using the standard solution resistance, very similar wave patterns were observed between $V_{\text {in }}$ and $V_{\text {out }}$.

the $R_{\text {sol }}$ values that were obtained in the actual animal experiment slightly differed in both latency and amplitude between the signal source potential $V_{\text {in }}$ and the output potential $V_{\text {out }}$ (input potential to the bioamplifier), as shown in Fig. 3. The emulation result based on the high $R_{\text {sol }}$ values had a longer latency and a decreased amplitude. 


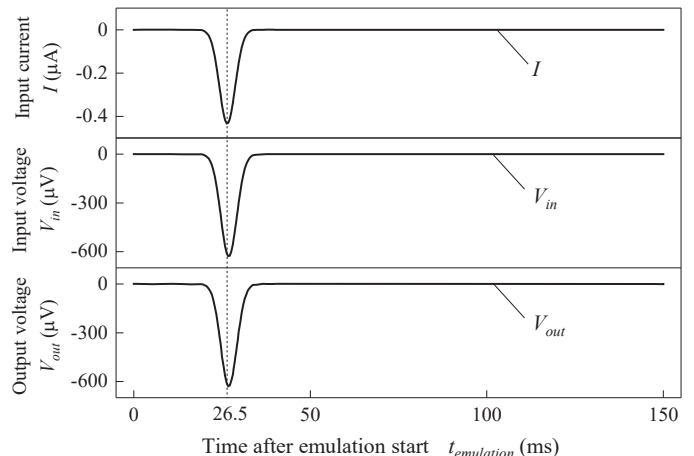

Fig. 15. Result of visual evoked potential emulation with the maximum solution resistance in 32 weeks in rabbit A. The input current $I$ is denoted in Eq. (3). The input voltage $V_{\text {in }}$ and the output voltage $V_{\text {out }}$ are as described in Fig. 2. By using the maximum solution resistance, very similar wave patterns were observed between $V_{\text {in }}$ and $V_{\text {out }}$.

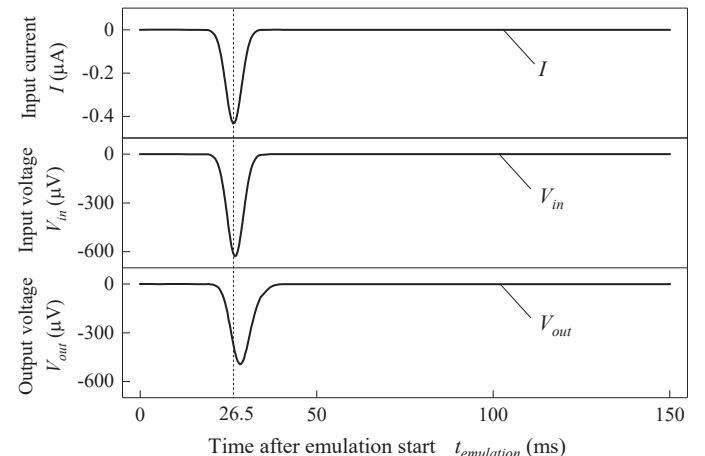

Fig. 16. Result of visual evoked potential emulation with the significantly higher solution resistance in rabbit A. The input current $I$ is denoted in Eq. (3). The input voltage $V_{\text {in }}$ and the output voltage $V_{\text {out }}$ are as described in Fig. 2. Increasing latency and decreasing amplitude were observed in the $V_{\text {out }}$ wave compared with that for the $V_{i n}$ wave.

\section{Discussion}

We used equivalent circuit analysis to demonstrate that $R_{s o l}$ values are altered in response to the tissue surrounding a recording electrode. The in vivo $R_{\text {sol }}$ was increased compared with the in vitro value due to electrode contact with living tissue. The time-dependent increase in $R_{\text {sol }}$ values was related to electrode surface condition changes that occurred in response to surgical damage, such as the formation of a fibrosis encapsulation layer around the electrode. ${ }^{(5)}$ Thus, we assumed that the initial $R_{c t}$ value decreased a few weeks after electrode implantation because of tissue fluid and blood infiltration; subsequently, $R_{c t}$ stabilized at a high value after the amount of tissue fluid infiltration decreased. The CPE approximates an electric double-layer capacitor and represents both the nonlinearity that results from the adsorption of proteins and the effects of surface roughness, in contrast to pure capacitance. ${ }^{(12)}$ The values of $n$ in Eq. (1) were close to 1. This finding suggests that the primary property of the CPE used in this study was capacitive. For the implanted electrode, the capacitance is dependent on the medium surrounding the electrode interface. The CPE admittance values were stable and almost unchanged compared with the in vitro values; thus, the effect of the medium surrounding the electrode interface was minimal. In addition, the $R_{c t}$ values after several weeks of electrode implantation were like those observed under the in vitro condition; thus, the in vivo electrode reactivity characteristics were considered stable. The inflammation and fibrosis of the tissue surrounding the electrodes were likely results of wound healing. Electrode implantation activates the host innate immune system to induce a foreign body reaction. This reaction involves protein adsorption on the electrode surface, inflammatory cell infiltration, macrophage fusion into foreign body giant cells, fibroblast activation, and ultimately fibrous encapsulation. Such a foreign body reaction at the interface of the electrode continues for 2-4 weeks after implantation and affects the life of the implanted device. ${ }^{(28)}$ After the foreign body reaction subsides, the electrode characteristics stabilize.

The time-dependent increase in solution resistance suggests a continuation of tissue encapsulation around the electrode surface. As mechanical stress, such as pressure and rubbing, 
accumulates over time with chronic implantation, granulation tissue composed of fibers and collagen encapsulated the electrode. Stable CPE admittance indicated that protein adsorption did not occur after the foreign body reaction subsided. The continuous increase in $R_{\text {sol }}$ suggests that the connective tissue thickened and grew outward, despite the stabilization of the electrode-surface $R_{c t}$ or CPE characteristics. In the equivalent circuit analysis of EIS, the $R_{c t}$ and CPE values differ for the electrode material. The electrode materials do not affect $R_{\text {sol }}$, as this characteristic reflects changes in the tissue surrounding the electrode. Recording electrode composed of biocompatible and capacitive characteristic materials other than Pt show a similar electrode surface interface behavior, such as the EIS time change.

The stability of the recording electrode characteristics was confirmed on the basis of the stability of the $\mathrm{N}_{1}$ amplitude of the VEP. The observed time-dependent decrease in the VEP latency is consistent with the results of previous reports. ${ }^{(29)}$ We emulated the evoked potentials to determine whether changes in the recording electrode characteristics affected the evoked potentials. The difference between the emulation reflecting the recording electrode characteristic change and the actual VEP change revealed the factor that changed the VEP. Thus, theoretically, VEP emulation can be used to determine the influence of other factors on VEP modulation, such as cell type, electrode interface, or bioamp and input impedance. We found that increases in solution resistance that are within the range of values obtained in actual animal experiments slightly affect the size and latency of evoked potentials. Thus, the time-dependent decrease in VEP latency was not due to changes in the recording electrode characteristics. Moreover, regardless of whether we used a different type of electrode (where the electrode materials were not $\mathrm{Pt}$ ), the $\mathrm{N}_{1}$ latency decreased. An evaluation of the $\mathrm{N}_{1}$ latency is useful for VEP assessment because $\mathrm{N}_{1}$ is a reliable reaction in cortex layer $\mathrm{IV}^{(30)}$ Our findings with a different electrode confirmed that a decreasing $\mathrm{N}_{1}$ latency occurs during chronic VEP measurement and that this decreased $\mathrm{N}_{1}$ latency is not due to changes in the characteristics of electrode interface, but to biological reactions. Moreover, using an electrode made of a material other than Pt would likely yield comparable results.

The specific reasons for the observed decrease in VEP latency are unknown; however, three possibilities should be considered. First, glial cells, which support neuronal signaling, might have affected our results. When brain tissue is damaged, astrocytes are stimulated to protect neurons via $\mathrm{Ca}^{2+}$ signaling, resulting in wound healing. ${ }^{(31)}$ In addition, astrocytes' $\mathrm{Ca}^{2+}$ is important for the regulation of synaptic function. ${ }^{(31)}$ We hypothesize that electrode implantation induced nerve damage, and subsequently, astrocytic neuronal protection and wound healing, altering synaptic transmission. As the result, animal's reactions improve gradually. Hence, $\mathrm{N}_{1}$ latency chronologically decreases. Second, our observations could have been due to electrodeimplantation-induced mechanical pressure on the brain, which decreased the distance to the signal source. This reduction in distance to the signal source can decrease latency. ${ }^{(32)}$ Third, the anesthesia we used may have affected the recorded evoked potentials. ${ }^{(33)}$ That is, the rabbits may have become accustomed to the isoflurane anesthesia due to repeated exposure. Although we measured the anesthetic concentrations in the exhaled gas, anesthesia acclimation could have decreased the depth of anesthesia. Monitoring of the bispectral index via EEG analysis, which is used to assess the level of consciousness during anesthesia, can be used to control the anesthesia depth and would resolve this issue. The emulation of the evoked potentials using an extremely large solution resistance increased latency and decreased amplitude. This finding suggests that greater damage to the brain following electrode implantation increases the foreign body reactions 
around the electrode, resulting in a marked increase in solution resistance. This idea is consistent with reports suggesting that conventional recording electrodes can only be used for the shortterm recording of evoked potentials. ${ }^{(2,7)}$ Our emulation approach can estimate recording electrode degradation. The time constant $\tau$ of the equivalent circuit for the electrode surface [the electrode interface component as shown in Fig. (3)] is based on $R_{s o l}, R_{c t}$, and $C_{d l}$, as represented in Eq. (4).

$$
\tau=\frac{R_{s o l} \times R_{c t} \times C_{d l}}{R_{s o l}+R_{c t}}
$$

Equation (4) shows that large increases in solution resistance increase $\tau$. Hence, our analysis indicates that solution resistance and the time constants chronologically increase as the reaction slows. A marked increase in solution resistance can degrade VEP recording; therefore, extreme elevations of solution resistance should be avoided for the long-term recording of evoked potentials.

\section{Conclusions}

We used EIS and equivalent circuit analysis to determine the long-term changes in the properties of recording electrodes. Our recording electrodes performed exceptionally well for long-term recording, as we did not observe an $\mathrm{N}_{1}$ amplitude reduction or an $\mathrm{N}_{1}$ latency extension over time. In a previous study, an $\mathrm{N}_{1}$ amplitude reduction and $\mathrm{N}_{1}$ latency extension occurred in response to foreign body reactions around the electrodes. ${ }^{(2)}$ Our VEP emulation results support these findings of changes in the VEP. Although we observed the decreased $\mathrm{N}_{1}$ latency, our emulation results suggest that this decrease in latency was not related to the electrode characteristics, but rather, to biological reactions in the electrode-implanted tissue. Nonetheless, our approach for equivalent circuit analysis and evoked potential emulation improved our understanding of the interaction between the electrode interface characteristics and the stimulation response. Our approach using VEP emulation was useful for determining whether electrode characteristics or biological reaction modulates the VEP and can be used further to identify the factors that affect time-dependent changes in the VEP. Moreover, our technique can be used for the long-term evaluation of novel electrodes in neural prostheses. In future studies, we will investigate the effects of glial cells and anesthesia depth on evoked potentials.

\section{Acknowledgments}

The authors thank Masayoshi Nakatani and Chisato Taki of Nidek Co., Ltd. for their assistance with the animal experiments. This study was supported by the New Energy and Industrial Technology Development Organization (NEDO) of Japan.

\section{References}

1 G. Márton, I. Bakos, Z. Fekete, I. Ulbert, and A. Pongrácz: J. Mater. Sci.-Mater. Med. 25 (2014) 931.

2 K. Makowiecki, A. Garrett, V. Clark, S. L. Graham, and J. Rodger: Transl. Vis. Sci. Technol. 4 (2015) 1.

3 A. A. Eshraghi, R. Nazarian, F. F. Telischi, S. M. Rajguru, E. Truy, and C. Gupta: Anat. Rec. 295 (2012) 1967.

4 S. F. Lempka, S. Miocinovic, M. D. Johnson, J. L. Vitek, and C. C. McIntyre: J. Neural. Eng. 6 (2009) 1.

5 A. Mercanzini, P. Colin, J. C. Bensadoun, A. Bertsch, and P. Renaud: IEEE Trans. Biomed. Eng. 56 (2009) 1909. 
6 T. Fujikado, M. Kamei, H. Sakaguchi, H. Kanda, T. Endo, M. Hirota, T. Morimoto, K. Nishida, H. Kishima, Y. Terasawa, K. Oosawa, M. Ozawa, and K. Nishida: Invest. Ophthalmol. Visual Sci. 57 (2016) 6147.

7 M. Kuwabara, H. Tashiro, Y. Terasawa, K. Osawa, T. Tokuda, J. Ohta, and T. Fujikado: Adv. Biomed. Eng. 6 (2017) 59.

8 T. Okuno, H. Oku, and T. Ikeda: Neuro-Ophthlmol. 26 (2002) 59.

9 K. Wang, X.-Q. Li, X.-X. Li, W.-H. Pei, H.-D. Chen, and J.-Q. Dong: Vision Res. 51 (2011) 1897.

10 I. Guarino, S. Loizzo, L. Lopez, A. Fadda, and A. Loizzo: Neural Plast. 11 (2004) 241.

11 J. Charng, C. T. Nguyen, Z. He, T. M. Dang, A. J. Vingrys, R. L. Fish, R. Gurrell, P. Brain, and B. V. Bui: PLoS One 8 (2013) e 74172.

12 R. de Levie: J. Electroanal. Chem. 261 (1989) 1.

13 B. E. Conway: Impedance Spectroscopy, eds. E. Baesoukov and J. R. Macdonald (Wiley, New Jersey, 2005) pp. $475-479$.

14 A. Prasad and J. C. Sanchez: J. Neural Eng. 9 (2012) 1.

15 M. E. Orazem and B. Tribollet: Electrochemical Impedance Spectroscopy (Wiley, New Jersey, 2008) Chap. 9.

16 X. F. Wei and W. M. Grill: J. Neural Eng. 6 (2009) 1.

17 V. Sankar, E. Patrick, R. Dieme, J. C. Sanchez, A. Prasad, and T. Nishida: Front. Neuroeng. 7 (2014) 1.

18 G. Rondelli, P. Torricelli, M. Fini, and R. Giardino: Biomaterials 26 (2005) 739.

19 D. D. Venuto, P. Ledochowitsch, M. Maharabitz, and J. Rabaey: Proc. ISQED (IEEE, Santa Clara, 2014) pp. 380-386.

20 N. Yousif, R. Bayford, and X. Liu: Neuroscience 156 (2008) 597.

21 M. A. Moffitt and C. C. McIntyre: Clin. Neurophysiol. 116 (2005) 2240.

22 C. H. Im, C. Lee, H. K. Jung, and S. Y. Lee: IEEE Trans. Magn. 44 (2008) 1434.

23 C. H. Im, H. K. Jung, and N. Fujimaki: Phys. Med. Biol. 50 (2005) 4931.

24 H. A. Shah, S. R. Montezuma, and J. F. Rizzo 3rd: Exp. Eye Res. 83 (2006) 247.

25 W. Franks, I. Schenker, P. Schmutz, and A. Hierlemann: IEEE Trans. Biomed. Eng. 52 (2005) 1295.

26 B. E. Conway: Impedance Spectroscopy, eds. E. Baesoukov and J. R. Macdonald (Wiley, New Jersey, 2005) p. 494.

27 M. Kuwabara, H. Tashiro, Y. Terasawa, Y. Nakano, Y. Yoshimura, K. Osawa, T. Noda, T. Tokuda, and J. Ohta: IEICE Res. Tech. Rep. 116 (2017) 25 (in Japanese).

28 J. M. Anderson, A. Rodriguez, and D. T. J. Chang: Semin Immunol. 20 (2008) 86.

29 T. Ondrejcák, I. Vanický, J. Gálik, and K. Saganová: J. Neurosci. Methods 141 (2005) 125.

30 L. B. Padnick and R. A. Linsenmeier: Vision Res. 39 (1999) 2833.

31 K. Kanemaru, J. Kubota, H. Sekiya, K. Hirose, Y. Okubo, and M. Iino: Proc. Natl. Acad. Sci. USA. 110 (2013) 11612.

32 G Buzsáki, C. A. Anastassiou, and C. Koch: Nat. Rev. Neurosci. 13 (2012) 407.

33 P. S. Sebel, D. A. Ingram, P. J. Flynn, C. F. Rutherfoord, and H. Rogers: Br. J. Anaesth. 58 (1986) 580. 